\title{
Extragalactic Planetary Nebulae as Mass Tracers: Observational Aspects
}

\author{
Magda Arnaboldi \\ INAF - National Institute for Astrophysics, Astronomical Observatory \\ of Capodimonte, Via Moiariello 16, I-80131 Naples
}

\begin{abstract}
Planetary nebulae observed in our Milky Way galaxy and in the galaxies of the Local Group do impress us for their morphologies and complexities. But when we look at planetary nebulae outside the Local Group, all this must be forgotten, as they become unresolved and merely points of green light. This paper will review how these green spots of light can be used as probes of the mass distribution and dynamics of elliptical galaxies and nearby clusters.
\end{abstract}

\section{Introduction}

From the early work by Nolthenius and Ford (1987), the use of of planetary nebulae $(\mathrm{PNe})$ as tracers of the stellar light, chemical composition and dynamical status has now established itself as an important topic in this field. In this paper we will review the techniques implemented to detected PNe in distant galaxies, the information we were able to obtain about the dynamical status of galaxy outer halos, and the recent discovery of PNe in clusters of galaxies, freeflying in the cluster space between galaxies. This field is also receiving greater inputs from the recent development of ad-hoc instrumentation as the Planetary Nebulae Spectrograph, which is described in the contribution from N. Douglas, this volume.

\section{PNe as probes of the dynamics of the galaxies outer halos}

Elliptical galaxies can be considered excellent laboratories to test the galaxy formation theories because secondary infall models of galaxy formation in CDM universes predict segregation of angular momentum in their outer regions (Zurek et al. 1988) and the ubiquitous presence of dark matter. Furthermore, these theories relate the so-called morphology-environment correlation, i.e. $80 \%$ of galaxies in clusters are early-type while $80 \%$ of field galaxies are spirals, with the properties of dark halos. Dark halos in clusters are deeper according to these models, and the protogalaxies forming in these halos will end up as early-types (Quinn \& Zurek 1988).

Tests to confirm these predictions are hampered by observational and theoretical problems. Elliptical surface brightness profiles decrease rapidly with radius, and radial velocity plus velocity dispersion profile measurements based 
on absorption line techniques are confined within the brighter central regions, i.e. within $2-3 R_{e}{ }^{12}$.

Studies based on absorption line profile shapes, have given strong constraints on the mass distributions of ellipticals out to two effective radii $\sim 2 R_{e}$ (Gerhard et al. 1998, Saglia et al. 2000, Kronawitter et al. 2000; see also Rix et al. 1997), with a small to moderate dark matter fraction required inside $2 R_{e}$ such that the total circular velocity curves are approximately flat (Gerhard et al. 2001). In giant elliptical galaxies, the stellar kinematics in these regions is dominated by (anisotropic) velocity dispersions, with rotation contributing only a smaller fraction of kinetic energy (contrary to small ellipticals, see Rix et al. 1999). These optical spectral studies are difficult to extend beyond radii of 2$3 R_{e}$. At larger radii (from where a sizable $30-40 \%$ of the galaxy light emanates) the starlight is simply too faint compared to the night sky level for deep spectral studies to be feasible. Moreover ellipticals do not have cold HI disks at large radii as in spiral galaxies: in these hot stellar systems we do not know the orbital properties of stellar motions, and therefore the relationship between intrinsic and projected quantities is uncertain.

Recent work in several fields, however, leaves no doubt about the existence of dark matter in ellipticals: X-ray data on their hot gas atmospheres imply that dark halos in ellipticals are ubiquitous and that the mass-to-light ratios are $M / L \sim 100$ on scales of $\sim 100 \mathrm{kpc}$ (Mushotzky et al. 1994, Matsushita et al. 1998, Loewenstein \& White 1999). This work will be greatly strengthened by the ongoing analysis of data from the Chandra and XMM satellites. Gravitational lensing studies (Keeton, Kochanek \& Falco 1998, Griffiths et al. 1998, Wilson et al. 2001) show evidence for large $M / L$ in lens elliptical galaxies.

Nevertheless, the outskirts of galaxies are important for understanding galaxy structure and formation. There the influence of the dark halo is greatest, and issues such as dark matter fractions in elliptical galaxies are most reliably addressed by measurement of the outermost stellar kinematics. The outer regions of galaxies are also those with the longest dynamical times, hence it is the place to look for non-equilibrium structures still associated with the formation of elliptical galaxies. High angular momentum material acquired late will not reach the brighter inner regions, but would instead show up in the outskirts.

To extend our work on elliptical galaxy halos to larger radii, a well-observable tracer of the outer parts of galaxies' stellar bodies is required, and is provided by planetary nebulae $(\mathrm{PNe})$. PNe are the final stages of intermediate, $1-8 \mathrm{M}_{\odot}$, mass stars, therefore in an evolved population like those observed in giant early-type galaxies several hundred $\mathrm{PNe}$ can be observed with 4 meter class telescopes in systems at a distance of 15-17 Mpc. These stars emit most of their light in a few strong emission lines, which may be detected at much higher contrast against the night sky than the continuum spectra of stars in other phases of evolution. Their simple spectra make radial velocity measurement straightforward. They have also a well-defined luminosity function which has been used with success as a tertiary distance indicator for elliptical and early-type disk galaxies. The luminosity function is characterized by a sharp cutoff at the bright end; the

${ }^{12} R_{e}$ is the effective radius which encompasses half of the total light in an E galaxy with an $R^{1 / 4}$ luminosity profile. 
number of PN with brightness at least one tenth the cutoff is around 1 per $10^{7}$ $B$-band luminosities. All the empirical evidences obtained so far indicate that PNe number density follow the stellar light, therefore they represent an excellent probe to study the dynamical propertied of the Es outer halos. At this Symposium, several poster papers were devoted to this topic and about one thousands radial velocities are measured for some of the nearest objects, i.e. NGC 5128.

\section{Best examples of what PNe can do for us}

The projected number density distribution and 2D radial velocity fields from $\mathrm{PNe}$ can be used to constrain the mass distribution and the angular momentum in the halos of early-type galaxies. The case of NGC 5128 (Hui et al. 1995) provide evidence for a triaxial halo and large rotation $(\mathrm{v} / \sigma \simeq 1)$ in the galaxy outer region. Recent developments are supporting these earlier findings, see E. Peng's contribution, this volume. Similar results were obtained for other two early-type galaxies, NGC 4406 and NGC 1316 (Arnaboldi et al. 1996, 1998), which displayed large rotation in the outer parts and an increasing $\mathrm{M} / \mathrm{L}$ ratio, of a factor 2 between the inner regions $R<1.5 \mathrm{kpc}$ and $R<15-20 \mathrm{kpc}$. On the other hand, there seems to be objects whose DM halo has been stripped: $R$. Méndez has recently measured 535 radial velocities of $\mathrm{PNe}$ in the flattened elliptical NGC 4697 (Méndez et al. 2001). The kinematics traced by the radial velocities of $\mathrm{PNe}$ is in agreement with the stellar rotation. In this object, the mass distribution seems best reproduced by a model with constant $\mathrm{M} / \mathrm{L}$ ratio $=$ 11 (see R. Méndez's contribution, this volume), with the rotation curve gently decreasing in the outer parts. Other early-type objects for which constant $\mathrm{M} / \mathrm{L}$ mass models were found are NGC 3379 (Ciardullo et al. 1993) and NGC 3384 (Tremblay et al. 1995).

\section{2D PNe radial velocity fields: how to handle them}

The observational findings obtained up to now with PNe radial velocity fields are based on limited statistical samples: questions may rise on the precision and accuracy of those quantities obtained from such samples, and whether the estimates on the galaxy mass content and angular momentum are reliable. In particular: are the simple 3-parameter functional forms used to fit small sample of $P N e$ radial velocities, i.e. rigid body rotation or flat rotation curve, introducing biases on the estimates of the kinematical quantities? How does the precision of an estimate depend on the sample size, taking into account the rotation and inclination of the system under study?

Napolitano et al. (2001) built spherical systems using Hernquist analytical model and with a cylindrical flat rotation curve; all the observational biases, high surface brightness in the galaxy core and field size, were also considered.

Napolitano et al. indicate that a robust estimated quantity derived when using simple 3-parameter functions is the line of maximum velocity gradient: limited statistical sample of PN radial velocities do show whether rotation is present. A bilinear fit to a $2 \mathrm{D}$ velocity sample from a system with a rotation curve as the one of Centaurus A does introduce a bias in the estimate of the velocity dispersion profile at large radii, see Figure 1. Such a bias correlates 

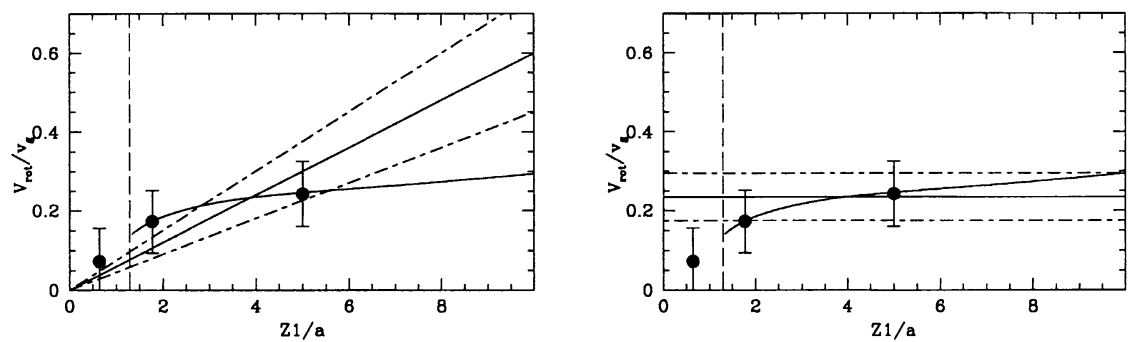

Figure 1. Biases in the maximum velocity gradient and maximum rotation velocity. The intrinsic rotation curve from the model (curved solid line) is compared with the bilinear fit (BF, left), the flat-curve fit ( $\mathrm{FC}$, right) and the mean values in bins (filled points) obtained on 100 simulations. The parameters of the fitted functions are the averages from the same 100 simulations: straight solid lines show the BF (left) and FC (right) fits, and dot-dashed lines are the SD lines about the fits. Quoted errors about the points are the SDs of the distribution of estimates in each bin. The dashed vertical line indicates the inner limiting radius.

with the maximum rotation velocity, as shown in Figure 2, and this problem is important and pervades more sophisticated non-parametric analysis, based on smoothing techniques. The overestimate in $\sigma$ caused by fitting a plane to the $2 \mathrm{D}$ radial velocity field can be up to $20 \%$ in this quantity, and $40 \%$ in the total mass.

Napolitano et al. were also able to give precise estimates of the relative errors on the velocity dispersion depending on the sample size: a sample size of 500 test particles give a precision of $16 \%, 23 \%$ for 150 particles, $25 \%$ for 50 test particles. Furthermore the precision obtained on a single measurement is consistent with those obtained from simulated distributions. The more accurate approach to descrete radial velocity fields is to perform the analysis of the binned quantities along the P.A. of the line of maximum gradient. When the best data are available, the rotation curve derived from binned quantities must be investigated in detail for any evidences of on-going interaction. Significant statistics based on extended rotation curves measured by coupling the HI $21 \mathrm{~cm}$ with optical emission line observations have disclosed a decline at large galactocentric distances, in contrast with the asymptotic flat behavior for field spirals (Rubin et al. 1999), and/or asymmetries. In the outer halos of early-type galaxies the standard approach is to assume equilibrium and invert the Jeans equations to derive the mass distribution. The case of NGC 1399 is a clear example: high $\mathrm{M} / \mathrm{L}$ ratios, about 60 , are obtained when equilibrium is assumed. Napolitano et al. (2002) have shown that under the non-equilibrium hypothesis a $\mathrm{M} / \mathrm{L}=$ 26 within $R=400^{\prime \prime}$ can reproduce the observed kinematics at radii of about 25 kpc. 


\section{PNe as tracers of cluster formation and evolution}

Diffuse light in clusters was first discovered by Zwicky in 1951 in his study of the galaxy luminosity function in the Coma cluster (Zwicky 1951). Several attempts followed, aiming at the detection of the total light in the diffuse component, which was hampered by the difficulty of detecting a surface brightness which is about $1 / 100$ of the night sky. An alternative to this method for probing intracluster light is through the direct detections of the stars themselves. In 1979 a search was done for intergalactic supernovae in the Coma cluster, but the outcome was negative. In 1981 a supernova Type Ia was discovered in the region between M86 and M84 in the Virgo cluster. In the eighties several studies argued for the pronounced globular cluster over-abundance of some galaxies at the center of galaxy clusters to be best explained by assuming the existence of a population of intergalactic globular clusters.

A new interest in these kind of studies was brought by the discovery of intracluster planetary nebulae free flying in the intracluster regions of Virgo and Fornax (Arnaboldi et al. 1996, Theuns \& Warren 1997, Ciardullo et al. 1998, Feldmeier et al. 1998), and the excess of red number counts in a field in Virgo with respect to the HDF (Ferguson et al. 1998, Durrell et al. 2002).

From the theoretical point of view, N-body simulations of star stripping from galaxies in clusters (Moore et al. 1996) due to fast speed encounters predicts that more than half of the mass of bright galaxies is lost in this way. If this is the way galaxies assemble to form a cluster, the number density of the ICPNe and their radial velocities can give information on the phase space distribution of this component and test this scenario for cluster formation. See the review by J. Feldmeier, this volume, for a complete discussion of the recent finding in Virgo and Fornax.

\section{Extragalactic Planetary Nebulae as tracers of the diffuse light in clusters}

We have begun a survey for IPN in the Virgo cluster to do dynamical studies and the selection of emission line candidates is based on the on-off technique developed by G. Jacoby and R. Ciardullo. It consists of acquiring an image of the field with a narrow band filter, centered on the strongest [OIII] $5007 \AA$ emission from a planetary nebula, redshifted at the PN redshift, and at the same time an off-band image is acquired with a filter centered on the [OIII] line adjacent continuum, or with a broad band large enough so that the contribution from the line to the total flux is negligible. Emission-line candidates were identified so far by-eye by blinking the on-and the off-band images, and retaining those objects which were visible in the on-band, but not in the off-band (Feldmeier et al. 1998, Ciardullo et al. 1998). The first spectroscopic follow-up (Kudritzki et al. 2000, Freeman et al. 2000) carried out on sub-samples of these catalogs showed that the on-off technique plus the "by-eye" identification of the PN candidates included some stars which were mis-classified as emission line objects. In addition, because of the very faint limiting fluxes reached by these surveys, they also detect high redshift emission-line objects, most probably from Ly $\alpha$ emitters at redshift $z=3.13$ and [OII] emitters at redshift $z=0.347$. Nearby 




Figure 2. Biases from BF in dark matter systems: points represent the relative deviation of the velocity dispersion determined in the last bin along Z1 with respect the "true" value expected from model. The behavior shown by the residuals indicates that higher rotation in the model causes the bilinear fit to introduce a larger bias in the estimated velocity dispersion profile.

clusters whose diffuse light can be traced by ICPNe cover large areas on the sky, and large mosaic of CCDs are needed. Both large areas and high angular resolution require the implementation of a robust automatic procedure to select a population of emission line candidates. Therefore it is necessary to i) identify a set of selection criteria based on the previous experience with the blinking technique, ii) tests these criteria on a spectroscopically selected sample of ICPNe and then iii) determine the fraction of high redshift emission line galaxies in these samples. The magnitude system for the [OIII] $5007 \AA$ emission was introduced by Jacoby $(1989)^{13}$. Our automatic technique (Arnaboldi et al. 2002), based on Theuns \& Warren (1997) effort, needs to compare fluxes in both the on and the off band, and therefore we need to work in $\mathrm{AB}$ magnitudes ${ }^{14}$. We used Sextractor (Bertin \& Arnouts 1996) for the photometry in V and narrow band. The onoff technique and tests of the spectroscopically confirmed sample translate in the following criteria for the identification of the best "emission line" ICPNe candidates:

(i) unresolved sources,

(ii) no detection in the off-filter at the position of a narrow band source,

(iii) sources with color $m_{v}-m_{n}<-1$.

It is important to point out that the $m_{v}-m_{n}<-1$ line is computed accounting for the photometric errors on the images, via the simulation of a point-like population.

When these selection criteria are implemented, the confirmation rate for a sample extracted from Feldmeier et al. (1998) field increase from $30 \%$ to $50 \%$, and the contamination from high redshift Ly $\alpha$ emitters at the bright end of the observed PN luminosity function (PNLF) is limited to $25 \%$ of the total sample, see Figure 3. This percentage of contamination from $\mathrm{L} \alpha$ emitters at redshifts

${ }^{13} m(5007)=-2.5 \log F_{5007}-13.74$ which gives the PN luminosity in equivalent $\mathrm{V}$ magnitude and $F_{5007}$ is the total flux in the line

${ }^{14} m_{A B}=-2.5 \log \left(F_{\nu}+19.436\right)$. The AB magnitude system may be considered as an instrumental magnitude, because it depends on the filter central wavelength, width and transmission. 


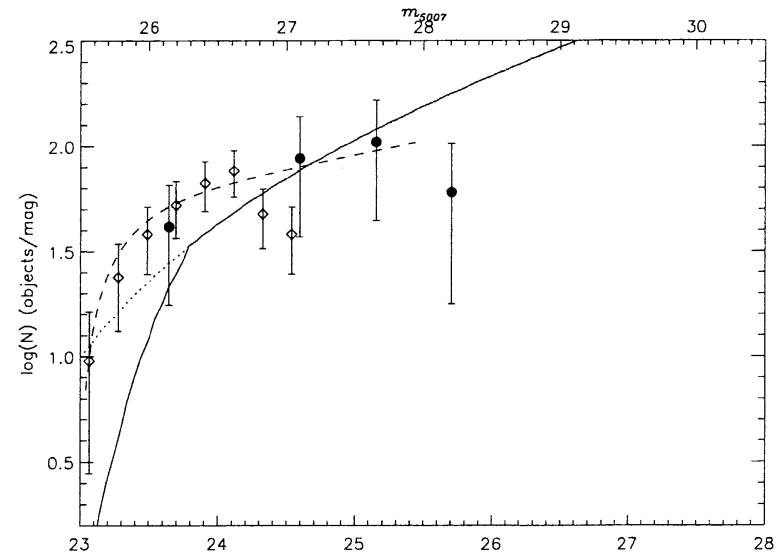

Figure 3. Luminosity function of the ICPNe sample from Arnaboldi et al. 2002. The dashed line shows the PNLF for a distance modulus of 30.31 , convolved with the photometric errors. On the same plot the continuous line shows the expected LF in our narrow filter for the Ly $\alpha$ population in the field at redshift $\mathrm{z}=3.13$ from Steidel et al. (2000). The faint dotted line shows the expected contribution from the Ly $\alpha$ emitters with V magnitudes brighter than 24.75. Full dots indicate the LF in our narrow band filter from Ly $\alpha$ emitters in the blank-field survey by Cowie \& $\mathrm{Hu}$ (1998).

$z=3.13$ were also independently confirmed by Ciardullo et al. (2002), based on narrow band surveys of blank fields.

When the contamination by high redshift galaxies is mastered, the PN luminosity function and the PN number density distribution can be used to trace the 3D shape of the nearby cluster and produce estimate of the percentage of total light in the diffuse component; see Feldmeier's review, this volume, for an detailed discussion of the uncertainties of these quantities.

\section{Conclusions}

The use of extragalactic PNe has now established itself as a main topic in the field of PN research. They can be used as tracers of the chemical properties of the stellar populations, as dynamical probes to establish the mass distribution and angular momentum distribution of galaxy outer halos. Furthermore the recent discovery of ICPNe, the efforts to perform surveys of large areas in nearby clusters, and techniques to reduce the contamination from high redshift interlopers make them reliable tools to test the theories for cluster formation and evolution.

\section{References}

Arnaboldi, M., et al. 1996, ApJ, 472, 145

Arnaboldi, M., et al. 1998, ApJ, 507, 759

Arnaboldi, M., et al. 2002, AJ, in press (astro-ph/0110522)

Bertin, E., \& Arnouts, S. 1996, A\&AS, 117, 399 
Ciardullo, R., et al. 1993, ApJ, 414, 454

Ciardullo, R., et al. 1998, ApJ, 492, 62

Ciardullo, R., et al., 2002, AJ, in press (astro-ph/0110456)

Cowie, L.L., \& Hu, E.M. 1998, AJ, 115, 1319

Durrell, P., et al. 2002, ApJ, in press (astro-ph/0201219)

Feldmeier, J.J., et al. 1998, ApJ, 503, 109

Ferguson, H., et al. 1998, Nature, 391, 461

Freeman, K., et al. 2000, Asp. Conf. Series, 197, 389

Gerhard, O.E., et al. 1998, MNRAS, 295, 197

Gerhard, O.E., et al. 2001, AJ, 121, 1936

Griffiths, R.E., et al. 1998, MNRAS, 282, 1159

Hui, X., et al. 1995, ApJ, 449, 592

Keeton, C.R., et al. 1998, ApJ, 509, 561

Kronawitter, A., et al. 2000, A\&AS, 144, 53

Kudritzki, R.-P., et al. 2000, ApJ, 536, 19

Jacoby, G. 1989, ApJ, 339, 39

Loewenstein, M., \& White, R.E. 1999, ApJ, 518, 50

Matsushita, K., et al. 1998, ApJ, 499, L13

Méndez, R., et al. 2001, ApJ, 563, 135

Moore, B., et al. 1996, Nature 379, 613

Mushotzky, R.F., et al. 1994, ApJ, 436, L79

Napolitano, N.R., et al. 2001, A\&A, 377, 784

Napolitano, N.R., et al. 2002, A\&A, in press (astroph/0112533)

Nolthenius, R., \& Ford, H. 1987, ApJ, 317, 62

Quinn, P., \& Zurek, W.H. 1988, ApJ, 331, 1

Rix, H.-W., et al. 1997, ApJ, 488, 702

Rix, H.-W., et al. 1999, ApJ, 513, L25

Rubin, V.C., et al. 1999, AJ, 118, 236

Saglia, R.P., et al. 2000, AJ, 119, 153

Steidel, C., et al., 2000, ApJ, 532, 170

Theuns, T. \& Warren, S. 1997, MNRAS, 284, L11

Tremblay, B., et al. 1995, ApJ, 443, L5

Wilson, G., et al. 2001, ApJ, 555, 572

Zwicky, F. 1951, PASP, 63, 61

Zurek, W.H., et al. 1988, ApJ, 330, 519 